\title{
ON SOME SUBSPACES OF BANACH SPACES WHOSE DUALS ARE $L_{1}$ SPACES
}

\section{ZIPPIN}

1. Introduction. Banach spaces whose duals are $L_{1}$ spaces were extensively studied in [3]. Recently J. Lindenstrauss and D. Wulbert [4] proved some results on the classification of these spaces which complemented the results of [3]. In [2] the results of [5] were applied to prove the following structure theorem for the separable case:

Proposition 1 [2, Theorem 2]. A separable Banach space $X$ satisfies $X^{*}=L_{1}$ space if and only if $X$ has a monotone basis $\left\{x_{i}\right\}_{i=1}^{\infty}$ such that for every $n$ the span of $\left\{x_{i}\right\}_{i=1}^{n}$ is isometric to $l_{\infty}^{n}$ (=the space of $n$-tuples $\lambda=\left(\lambda_{1}, \lambda_{2}, \cdots, \lambda_{n}\right)$ of reals with $\left.\|\lambda\|=\max _{1 \leqq i \leqq n}\left|\lambda_{i}\right|\right)$.

We recall that a sequence $\left\{x_{i}\right\}_{i=1}^{\infty}$ in a Banach space $X$ is called a monotone basis of $X$ (see $[1$, p. 67]) if each $x \in X$ has a unique representation $x=\sum_{i=1}^{\infty} \alpha_{i} x_{i}$ where $\left\{\alpha_{i}\right\}$ are scalars and the projections $P_{n}$ on $X$ defined by $P_{n}\left(\sum_{i=1}^{\infty} \alpha_{i} x_{i}\right)=\sum_{i=1}^{n} \alpha_{i} x_{i}$ are of norm 1 .

The purpose of this note is to show that the space $c_{0}$ (= the space of real sequences $\rho=\left\{\rho_{n}\right\}_{h=1}^{\infty}$ which converge to 0 with $\left.\|\rho\|=\sup _{1 \leqq n}\left|\rho_{n}\right|\right)$ is the minimal infinite dimensional Banach space whose dual is an $L_{1}$ space, namely

THEOREM 1. Let $X$ be a separable infinite dimensional Banach space whose dual is an $L_{1}$ space. Then $X$ has a subspace $V$ isometric to $c_{0}$ such that there is a projection of norm 1 from $X$ onto $V$.

It is proved in $[3$, p. 67 , Corollary 2] that any infinite dimensional Banach space $Y$ with $Y^{*}=L_{1}(\mu)$ has a separable infinite dimensional subspace $X$ for which $X^{*}=L_{1}(\nu)$. Hence, Theorem 1 implies the following

COROLlARY 1. Every infinite dimensional Banach space whose dual is an $L_{1}$ space contains a subspace isometric to $c_{0}$.

2. Preliminary lemmas. Let $X$ be a separable Banach space such that $X^{*}$ is an $L_{1}$ space and assume that $\left\{x_{i}\right\}_{i=1}^{\infty}$ is the monotone basis of $X$ mentioned in Proposition 1. It follows from Proposition 1 that in each subspace $E_{n}=\operatorname{span}\left\{x_{i}\right\}_{i=1}^{n}$ there is a basis $\left\{e_{i}^{n}\right\}_{i=1}^{n}$ such that

$$
\left\|\sum_{i=1}^{n} \gamma_{i} e_{i}^{n}\right\|=\max _{1 \leqq i \leqq n}\left|\gamma_{i}\right|
$$

Received by the editors March 19, 1969. 
for any real $\gamma_{1}, \gamma_{2}, \cdots, \gamma_{n}$. It is easy to prove (see $[4, \S 5]$ and $[5]$ ) that the bases $\left\{e_{i}^{n}\right\}_{i=1}^{n}, n=1,2,3, \cdots$ can be chosen such that for every $n$ and $1 \leqq i \leqq n$

$$
e_{i}^{n}=e_{i}^{n+1}+a_{i}^{n} e_{n+1}^{n+1}
$$

where $\sum_{i=1}^{n}\left|a_{i}^{n}\right| \leqq 1$. We define now a sequence $\left\{\phi_{i}\right\}_{i=1}^{\infty}$ of functionals on $\cup_{n=1}^{\infty} E_{n}$ in the following way: For each $j<n$ and $x=\sum_{i=1}^{n} b_{i} e_{i}^{n}$, $\phi_{j}(x)=b_{j}$. Using (2) it is easy to show that $\phi_{j}$ is uniquely defined and linear. Moreover, $\left|\phi_{j}(x)\right|=\left|b_{j}\right| \leqq \max _{1 \leqq i \leqq n}\left|b_{i}\right|=\|x\|$ and hence, $\phi_{j}$ can be extended by continuity to a linear functional of norm 1 on $X$. Another property of $\phi_{j}$ is proved in the following

Lemma 1. For each $j \phi_{j}$ is an extreme point of the unit ball of $X^{*}$.

Proof. Assume that $\phi_{j}=1 / 2(f+g)$ where $f, g \in X^{*}$ and $\|f\|=\|g\|$ $=1$. For all $n \geqq j, 1=f\left(e_{j}^{n}\right)=g\left(e_{j}^{n}\right)$ and if, for some $k \neq j, f\left(e_{k}^{n}\right)=\alpha \neq 0$ then

$$
f\left(e_{j}^{n}+(\operatorname{sign} \alpha) e_{k}^{n}\right)=1+|\alpha|>1 .
$$

This contradicts the fact that $\|f\|=1$ (since $\left.\left\|e_{j}^{n}+(\operatorname{sign} \alpha) e_{k}^{n}\right\|=1\right)$. It follows that $f\left(e_{\boldsymbol{k}}^{n}\right)=0$ for every $n \geqq j$ and $k \neq j$. Obviously $g$ shares the same property and therefore $f(x)=g(x)=\phi_{j}(x)$ for any $x \in \bigcup_{n=1}^{\infty} E_{n}$. Since $\bigcup_{n=1}^{\infty} E_{n}$ is dense in $X$ Lemma 1 is thus proved.

CoROllaRy 2. The closed linear span of $\left\{\phi_{j}\right\}_{j=1}^{\infty}$ is isometric to $l_{1}$.

Proof. $X^{*}$ is an $L_{1}(\mu)$ space and, as is well known, the extreme points of the unit ball are elements of the form $\phi=\chi_{A} / \mu(A)$ where $A$ is an atom and $\chi_{A}$ the characteristic function of $A$. It follows from Lemma 1 that $\left\|\sum_{i=1}^{\infty} \alpha_{i} \phi_{i}\right\|=\sum_{i=1}^{\infty}\left|\alpha_{i}\right|$ for any sequence $\left\{\alpha_{i}\right\}_{i=1}^{\infty}$ of reals. This concludes the proof.

For every $x=\sum_{j=1}^{n} b_{j} e_{j}^{n} \in \bigcup_{k=1}^{\infty} E_{k}$ we have $\|x\|=\max _{1 \leqq j \leqq n}\left|b_{j}\right|$ $=\max _{1 \leqq j \leqq n}\left|\phi_{j}(x)\right| \leqq \sup _{1 \leqq j<\infty}\left|\phi_{j}(x)\right| \leqq\|x\|$. It follows that for every $x \in X$

$$
\|x\|=\sup _{1 \leqq j<\infty}\left|\phi_{j}(x)\right| .
$$

The functionals $\phi_{j}$ will play an important role in the sequel.

LEMMA 2. Let $n(k)$ and $i(k)$ be increasing sequences of positive integers with $i(k) \leqq n(k)$. Then the sequence $\left\{e_{i(k)}^{n(k)}\right\}_{k=1}^{\infty}$ forms a monotone basis in the subspace $E$ which it spans in $X$. Moreover, in each subspace $F_{m}$ $=\operatorname{span}\left\{e_{i(\mathbf{k})}^{n(\boldsymbol{k})}\right\}_{\boldsymbol{k}=1}^{m}$ there is a basis $\left\{u_{i}^{m}\right\}_{i=1}^{m}$ satisfying the following

(a) $u_{m}^{m}=e_{i(m)}^{n(m)}$.

(b) For every $m$ and $i \leqq m, u_{i}^{m}=u_{t}^{m+i}+b_{i}^{m} u_{m+1}^{m+1}$ where $\sum_{i=1}^{m}\left|b_{i}^{m}\right| \leqq 1$. 
(c) $\left\|\sum_{i=1}^{m} \gamma_{i} u_{i}^{m}\right\|=\max _{1 \leqq i \leqq n}\left|\gamma_{i}\right|$ for any real $\gamma_{1}, \cdots, \gamma_{m}$.

Proof. Define $u_{1}^{1}=e_{i(1)}^{n(1)}$ and for $k>1$

$$
\begin{array}{rlrl}
u_{h}^{k} & =e_{i(k)}^{n(k)} & & \text { if } h=k \\
& =\sum_{j=1, j \neq i(k)}^{n(k)} b_{h, j}^{k} e_{j}^{n(k)} & \text { if } h<k \text { and } \quad u_{h}^{k-1}=\sum_{j=1}^{n(k)} b_{h, j}^{k} e_{j}^{n(k)} .
\end{array}
$$

The properties (a) and (b) follow directly from the definition of $u_{j}^{k}$ and from (c), while the following assertion is an easy consequence of (2): Let $x=\sum_{i=1}^{r} a_{i} e_{i}^{r}=\sum_{i=1}^{s} b_{i} e_{i}^{s}$ where $s>r$. Then $a_{i}=b_{i}$ for all $i \leqq r$. (Proof by induction on $s-r$.) In view of this fact and the definition of $u_{j}^{k}$ we have the equality $1=b_{j, i(j)}^{k}$ for $1 \leqq j<k$. Assertion (c) holds for $m=1$ and, assuming its validity for $m=k-1$, we get that $\sum_{h=1}^{k-1}\left|b_{h, j}^{k}\right| \leqq 1$ for all $1 \leqq j \leqq n(k)$. (This follows from the fact that $\left\{e_{j}^{n(k)}\right\}_{j=1}^{n(k)}$ is the usual basis in the $l_{\infty}^{n(k)}$ space $E_{n(k)}$ and that $\| \sum_{j=1}^{k-1}$ $\pm u_{j}^{k-1} \|=1$ for any choice of the signs.) In particular it follows that $b_{p, i(j)}^{k}=0$ for all $p \neq j, 1 \leqq p, j \leqq k$. We thus get that

$$
\max _{1 \leqq j \leqq k-1}\left|\gamma_{j}\right| \leqq\left\|\sum_{j=1}^{k-1} \gamma_{j} u_{j}^{k}\right\| \leqq\left\|\sum_{j=1}^{k-1} \gamma_{j} u_{j}^{k-1}\right\|=\max _{1 \leqq j \leqq k-1}\left|\gamma_{j}\right|
$$

for any real $\gamma_{1}, \cdots, \gamma_{k-1}$. The definition of $u_{k}^{k}$ and the last inequality yield

$$
\begin{aligned}
& \left\|\sum_{j=1}^{k} \gamma_{j} u_{j}^{k}\right\|=\left\|\sum_{j=1}^{k-1} \gamma_{j} u_{j}^{k}+\gamma_{k} e_{i(k)}^{n(k)}\right\| \\
& =\max \left\{\left\|\sum_{j=1}^{k-1} \gamma_{j} u_{j}^{k}\right\|,\left|\gamma_{k}\right|\right\}=\max _{1 \leqq j \leqq k}\left|\gamma_{j}\right| .
\end{aligned}
$$

This proves (c).

Now, for any sequence $\left\{\gamma_{j}\right\}_{j=1}^{k+1}$ of reals let $\sum_{j=1}^{k} \gamma_{j} e_{i(j)}^{n(f)}=\sum_{j=1}^{k} \beta_{j} u_{j}^{k}$. Then by (a), (b) and (c)

$$
\begin{aligned}
\left\|\sum_{j=1}^{k} \gamma_{j} e_{i(j)}^{n(j)}\right\| & =\max _{1 \leqq j \leqq k}\left|\beta_{j}\right| \\
& \leqq \max \left\{\max _{1 \leqq j \leqq k}\left|\beta_{j}\right|,\left|\gamma_{k+1}+\sum_{j=1}^{k} \beta_{j} b_{j}^{k}\right|\right\} \\
& =\left\|\sum_{j=1}^{k} \beta_{j} u_{j}^{k}+\gamma_{k+1} u_{k+1}^{k+1}\right\| \\
& =\left\|\sum_{j=1}^{k+1} \gamma_{j}^{j} u_{j}^{j}\right\|=\left\|\sum_{j=1}^{k+1} \gamma_{j} e_{i(j)}^{n(j)}\right\|
\end{aligned}
$$


This inequality shows that $\left\{e_{i(j)}^{n(f)}\right\}_{j=1}^{\infty}$ is a monotone basis of $E$ (see $[1$, p. 67$])$, and Lemma 2 is proved.

By Proposition 1 and Lemma 2 any sequence $\left\{e_{i(k)}^{n(k)}\right\}$, where $n(k)$ and $i(k)$ are increasing and $i(k) \leqq n(k)$, span a subspace $E$ whose dual is an $L_{1}$ space. As in the definition of the functionals $\left\{\phi_{j}\right\}$ given at the beginning of $\S 2$, we define functionals $\left\{\psi_{j}\right\}$ on $E$ by $\psi_{j}\left(\sum_{i=1}^{k} b_{i} u_{i}^{k}\right)$ $=b_{j}$ if $k \geqq j$ and then extend them by continuity to $E$. By (3) we have, for every $x \in E,\|x\|=\sup _{1 \leq j<\infty}\left|\psi_{j}(x)\right|$. Since $u_{h}^{k}=\sum_{j=1}^{n(\boldsymbol{k})} b_{h, j}^{k} e_{j}^{n(k)}$ where $b_{h, i(j)}^{k}=\delta_{h, j}$ we get that $\phi_{i(j)}\left(u_{h}^{k}\right)=\delta_{h, j}=\psi_{j}\left(u_{h}^{k}\right)$ for $1 \leqq j, h \leqq k$. Hence $\psi_{j}(x)=\phi_{i(j)}(x)$ for all $x \in E$ and thus

$$
\|x\|=\sup _{j}\left|\phi_{i(j)}(x)\right| \text {. }
$$

In the sequel we will need the following well known

LEMMA 3. Let $\left\{w_{k}\right\}_{k=1}^{\infty}$ be a sequence of elements of $c_{0}$ where $w_{k}$ $=\left(\omega_{1}^{k}, \omega_{2}^{k}, \cdots\right)$ and assume that the following conditions are satisfied:

$$
\begin{gathered}
\omega_{k}^{k}=1 \quad \text { for every } k \\
\sum_{k=1 ; k \neq i}^{\infty}\left|\begin{array}{c}
k \\
\omega_{i}
\end{array}\right| \leqq 1 / 2 .
\end{gathered}
$$

Then $c_{0}=\operatorname{span}\left\{w_{k}\right\}_{k=1}^{\infty}$.

PRoof. Let $U$ be the operator defined by $U\left(\sum_{k=1}^{\infty} \gamma_{k} e_{k}\right)=\sum_{k=1}^{\infty} \gamma_{k} w_{k}$ where $\left\{e_{k}\right\}_{k=1}^{\infty}$ denotes the usual unit vector basis in $c_{0}$. Since $U$ is given by the matrix $\left|\omega_{n}^{k}\right|$, we get by (6) and (7) that $\|I-U\| \leqq 1 / 2$, where $I$ is the identity on $c_{0}$. It follows that $U$ is invertible and hence $c_{0}=\operatorname{span}\left\{w_{k}\right\}_{k=1}^{\infty}$.

3. Proof of Theorem 1. Let $X$ be a separable Banach space whose dual is an $L_{1}$ space. By Proposition 1 there is a system $\left\{e_{i}^{n}\right\}_{i=1}^{n} n$ $=1,2, \cdots$ of bases of the type described in the beginning of $\$ 2$. Consider the functionals $\left\{\phi_{j}\right\}_{j=1}^{\infty}$ constructed there; by the $\omega^{*}$ sequential compactness of the unit ball in $X^{*}$ there is a subsequence $\left\{\phi_{n(j)}\right\}_{j=1}^{\infty}$ of $\left\{\phi_{n}\right\}_{n=1}^{\infty}$ which converges $\omega^{*}$ to a functional $\phi$. By Lemma 2 , the subspace $E=\operatorname{span}\left\{e_{n(\mathcal{N})}^{n(f)}\right\}_{j=1}^{\infty}$ has an $L_{1}$ space as a dual and if $\psi_{j}$ and $\psi$ denote the restrictions of $\phi_{n(j)}$ and $\phi$ to $E$ respectively, the following conditions are satisfied:

$$
\begin{gathered}
\|x\|=\sup _{1 \leqq j}\left|\psi_{j}(x)\right|, \\
\lim _{j \rightarrow \infty} \psi_{j}(x)=\psi(x),
\end{gathered}
$$


for every $x \in E$. Construct now in $E$ the bases $\left\{u_{j}^{\boldsymbol{k}}\right\}_{j=1}^{\boldsymbol{k}} k=1,2, \cdots$ satisfying (a), (b) and (c) of Lemma 2. It follows from (c) that for any choice of the signs $\left\|\sum_{i=1}^{k} \pm u_{i}^{k}\right\|=1$ and therefore, for any $k$ we have

$$
\sum_{i=1}^{k}\left|\psi\left(u_{i}^{k}\right)\right| \leqq 1
$$

Put $p(1)=16$ and choose $q(1), 1 \leqq q(1) \leqq 16$, such that $\left|\psi\left(u_{q(1)}^{p(1)}\right)\right|$ $\leqq 16^{-1}$. Denote $\mu_{1}=\psi\left(u_{q(1)}^{p(1)}\right)$ and choose

$$
\begin{array}{rlrl}
p(2) & \geqq 4 \cdot 8^{2} & \text { if } \mu_{1}=0 \\
\geqq 32^{-1} \mu_{1}^{-1} & \text { if } \mu_{1} \neq 0
\end{array}
$$

so large that

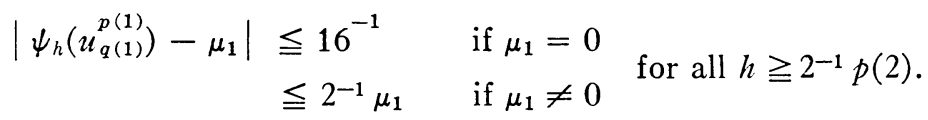

By (10) we can choose $q(2), 2^{-1} p(2) \leqq q(2) \leqq p(2)$, such that

$$
\begin{aligned}
\left|\psi\left(u_{q(2)}^{p(2)}\right)\right| & \leqq 2^{-1} \cdot 8^{-2} & & \text { if } \mu_{1}=0 \\
& \leqq 2^{-1} \cdot 8^{-1} \mu_{1} & & \text { if } \mu_{1} \neq 0 .
\end{aligned}
$$

Put $\mu_{2}=\psi\left(u_{q(2)}^{p(2)}\right)$. Proceeding by induction we define two increasing sequences $p(k)$ and $q(k)$ of positive integers such that if $\mu_{k}$ denotes $\psi\left(u_{q(k)}^{p(k)}\right)=\lim _{j \rightarrow \infty} \psi_{j}\left(u_{q(k)}^{p(k)}\right)$ then the following inequalities are satisfied:

$$
\begin{array}{rlrl}
\left|\mu_{k}\right| & \leqq 2^{-1} \cdot 8^{-k} \quad \text { if } \mu_{k-1}=0 \\
& \leqq 32^{-1} \mu_{k-1} \quad \text { if } \mu_{k-1} \neq 0 \\
\left|\psi_{h}\left(u_{q(k)}^{p(k)}\right)-\mu_{k}\right| & \leqq 2^{-1} \cdot 8^{-k} & \text { if } \mu_{k-1}=0 \\
& \leqq 2^{-1} \mu_{k-1} \quad \text { if } \mu_{k-1} \neq 0
\end{array}
$$

for all $h \geqq 2^{-1} p(k+1)$.

$$
p(k+1) \geqq q(k+1) \geqq 2^{-1} p(k+1)>p(k) .
$$

Now, either (I) there is an $N$ such that $\mu_{k} \neq 0$ for all $k>N$, or (II) there exists a sequence $\{i(k)\}_{k=1}^{\infty}$ of positive in tegers such that $\mu_{i(k)}=0$ for all $k$. Suppose that (I) holds, then we define for $k>N$

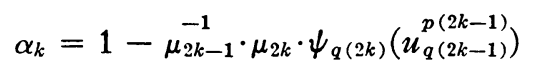

and 


$$
v_{k}=\alpha_{k}^{-1} \cdot\left(u_{q(2 k)}^{p(2 k)}-\mu_{2 k-1}^{-1} \cdot \mu_{2 k} \cdot u_{q(2 k-1)}^{p(2 k-1)}\right) .
$$

Using (11) and (12) one can easily prove that the elements $v_{k}$ of $E$ satisfy the following conditions:

$$
\begin{aligned}
& \lim _{j \rightarrow \infty} \psi_{j}\left(v_{k}\right)=0, \\
& \psi_{q(2 k)}\left(v_{k}\right)=1 \text {, } \\
& \left|\psi_{q(i)}\left(v_{k}\right)\right| \leqq 3 \cdot 2^{-1} \cdot 8^{-2 k} \quad \text { if } i>2 k \\
& \leqq 7^{-1} \quad \text { if } i=2 k-1 \\
& \leqq 0 \quad \text { if } i<2 k-1 \text {. }
\end{aligned}
$$

It follows from (16) that

$$
\begin{aligned}
& \sum_{k=1}^{\infty}\left|\psi_{q(2 n-1)}\left(v_{k}\right)\right| \leqq 1 / 4 \\
& \sum_{k=1 ; k \neq n}^{\infty}\left|\psi_{q(2 n)}\left(v_{k}\right)\right| \leqq 1 / 4
\end{aligned} \quad \text { for } n=1,2, \cdots .
$$

We apply now (4) and Lemma 2 to the space $E=\operatorname{span}\left\{u_{k}^{k}\right\}_{k=1}^{\infty}$ where $\left\{u_{i}^{\boldsymbol{k}}\right\}_{i=1}^{k}, \psi_{k}, p(k)$ and $q(k)$ play the role of $\left\{e_{i}^{\boldsymbol{k}}\right\}_{i=1}^{k}, \phi_{k}, n(k)$, and $i(k)$ of Lemma 2, respectively, and we get that for each $u \in U$ $=\operatorname{span}\left\{u_{q(k)}^{p(k)}\right\}_{k=1}^{\infty}, \quad\|u\|=\sup _{1 \leqq k}\left|\psi_{q(k)}(u)\right|$. We claim that if $v \in V_{0}$ $=\operatorname{span}\left\{v_{k}\right\}_{k=1}^{\infty}$ then $\|v\|=\sup _{1 \leqq k}\left|\psi_{q(2 k)}(v)\right|$. Indeed, if $v=\sum_{i=1}^{n} \lambda_{i} v_{i}$ and $\left|\lambda_{s}\right|=\max _{1 \leqq i \leqq n}\left|\lambda_{i}\right|$ for some $1 \leqq s \leqq n$ then by (17), for any $k$, $\psi_{q(2 k-1)}(v) \leqq \frac{1}{4}\left|\lambda_{s}\right|$ while $\left|\psi_{q(28)}(v)\right| \geqq 3 / 4\left|\lambda_{s}\right|$, and this proves the assertion. Define now a sequence $\left\{w_{k}\right\}_{k=1}^{\infty}$ in $c_{0}$ by $w_{k}=\left(\psi_{q(2)}\left(v_{k}\right), \psi_{q(4)}\left(v_{k}\right)\right.$, $\left.\psi_{q(6)}\left(v_{k}\right), \cdots\right)$. Our discussion above shows that the subspace $V_{0}$ $=\operatorname{span}\left\{v_{k}\right\}_{k=1}^{\infty}$ of $X$ is isometric to the subspace $W=\operatorname{span}\left\{w_{k}\right\}_{k=1}^{\infty}$ of $c_{0}$ (the transformation $T: V_{0} \rightarrow W$ defined by $T\left(\sum_{1}^{n} \gamma_{i} v_{i}\right)=\sum_{i=1}^{n} \gamma_{i} w_{i}$ for any $\sum_{i=1}^{n} \gamma_{i} v_{i} \in V_{0}$ can be extended to the desired isometry). The sequence $\left\{w_{k}\right\}_{k=1}^{\infty}$ satisfies, by (15) and (17), the assumptions of Lemma 3 and hence $W=c_{0}$. It is easy to see that (15), (16), and (17) yield the existence of a sequence $\left\{y_{k}\right\}_{k=1}^{\infty}$ in $V_{0}$ such that $\psi_{q(2 j)}\left(y_{k}\right)$ $=\delta_{j, k}, k, j=1,2, \cdots$. Consider the sequence $\left\{y_{2 k-1}-y_{2 k}\right\}_{k=1}^{\infty}$; obviously it spans in $V_{0}$ a subspace $V$ isometric to $c_{0}$ and

$$
\left\|\sum_{k=1}^{\infty} \lambda_{k}\left(y_{2 k-1}-y_{2 k}\right)\right\|=\max _{1 \leqq \boldsymbol{k}}\left|\lambda_{k}\right|
$$

for any sequence $\left\{\lambda_{k}\right\}$ in $c_{0}$. We note that $\psi_{j}$ is defined to be the restriction of $\phi_{n(j)}$ to $E$ and that $\{n(j)\}$ are chosen such that $\lim _{j \rightarrow \infty} \phi_{n(j)}(x)$ exists for all $x \in X$. Put $\bar{\phi}_{j}=\phi_{n(q(2 j))}$ and define for every $x \in X$ 


$$
P x=2^{-1} \sum_{j=1}^{\infty}\left(\bar{\phi}_{2 j-1}(x)-\bar{\phi}_{2 j}(x)\right)\left(y_{2 j-1}-y_{2 j}\right) .
$$

The transformation $P$ is obviously a projection from $X$ on to $V$ since $\lim _{j \rightarrow \infty}\left(\phi_{2 j-1}(x)-\phi_{2 j}(x)\right)=0$ for all $x \in X$. Moreover, $\|P x\|$ $=2^{-1} \sup _{1 \leqq j}\left|\bar{\phi}_{2 j-1}(x)-\bar{\phi}_{2 j}(x)\right| \leqq\|x\|$ by Corollary 2, and hence $\|P\|=1$.

It remains to consider the possibility (II). If (II) holds, we define $v_{k}=u_{q(i(k)))}^{p(i(k))}$ and $w_{k}=\left(\psi_{q(i(1))}\left(v_{k}\right), \psi_{q(i(2))}\left(v_{k}\right), \cdots\right)$ in $c_{0}$. Again, let $W=\operatorname{span}\left\{w_{k}\right\}_{k=1}^{\infty}$ and $V_{0}=\operatorname{span}\left\{v_{k}\right\}_{k=1}^{\infty}$, it is easy to show that $V_{0}$ and $W$ are isometric and that the sequence $\left\{w_{k}\right\}$ satisfies the assumptions of Lemma 3, and hence $V_{0}$ is isometric to $c_{0}$. The construction of $V$ and $P$ is done exactly in the same way. This proves Theorem 1.

4. Concluding remarks. A. Let $X$ be a separable Banach space whose dual is an $L_{1}$ space and assume that the unit cell of $X$ has at least one extreme point. Then $X$ contains a subspace $V$ isometric to $c$ ( $=$ the space of convergent sequences $\lambda=\left\{\lambda_{n}\right\}$ of reals with $\|\lambda\|$ $\left.=\sup _{n}\left|\lambda_{n}\right|\right)$ such that there is a projection of norm 1 from $X$ onto $V$. This assertion follows from the following considerations (which are only a sketch of the proof): It was observed by Semadeni [6] that a space satisfying the assumptions of our assertion is isometric to a space $Q(s)$ of affine continuous functions on a Choquet simplex $s$. Using the methods of [5] one can show that if $e$ denotes the unit function on $s$ then the bases $\left\{e_{i}^{n}\right\}_{i=1}^{n} n=1,2, \ldots$ can be chosen such that $e=e_{1}^{1}$ and $\bar{\phi}_{j}(e)=1$ for all $j$. The proof of Theorem 1 now shows that the subspace $V$ spanned by $\left\{y_{k}\right\}_{k=1}^{\infty}$ and $e_{1}^{1}$ in $X$ is isometric to $c$. Thedesired projection isgiven by $P(x)=\phi(x) e_{1}^{1}+\sum_{j=1}^{\infty}\left(\bar{\phi}_{j}(x)-\phi(x)\right) y_{j}$.

B. Given a sequence $\{m(i)\}_{i=1}^{\infty}$ of positive integers, the space $X$ and the system $\left\{e_{i}^{n}\right\}_{i=1}^{n}$ of bases described at the beginning of $\S 2$ we define $x_{i}=e_{i}^{s(k)}$ if $s(k-1)<i \leqq s(k)$, where $s(0)=0$ and $s(k)=\sum_{i=1}^{k} m(i)$. The sequence $\left\{x_{i}\right\}_{i=1}^{\infty}$ satisfies the property that

$$
\left\|\sum_{i=s(k)+1}^{s(k+1)} \lambda_{i} x_{i}\right\|=\max _{s(\mathbf{k})<i \leqq s(\boldsymbol{k}+1)}\left|\lambda_{i}\right|
$$

and by the proof of Lemma 2 it forms a basis in $X$. Call the bases $\left\{x_{n}\right\}$ and $\left\{y_{n}\right\}$ equivalent if the convergence of $\sum_{i=1}^{\infty} \alpha_{i} x_{i}$ is equivalent to the convergence of $\sum_{i=1}^{\infty} \alpha_{i} y_{i}$ for any real sequence $\left\{\alpha_{i}\right\}_{i=1}^{\infty}$. It follows that if the basis $\left\{e_{\boldsymbol{k}}^{k}\right\}_{\boldsymbol{k}=1}^{\infty}$ of $X$ is not equivalent to the unit vector basis of $c_{0}$ then by choosing the sequence $\{m(i)\}$ suitably one can construct infinitely many mutually nonequivalent bases of the form $\left\{x_{i}\right\}_{i=1}^{\infty}$. 


\section{REFERENCES}

1. M. M. Day, Normed linear spaces, Springer-Verlag, Berlin, 1962.

2. A. Lazar and J. Lindenstrass, On Banach spaces whose duals are $L_{1}$ spaces, Israel J. Math. 4 (1966), 205-207.

3. J. Lindenstrass, Extension of compact operators, Mem. Amer. Math. Soc. No. 48 (1964).

4. J. Lindenstrass and D. Wulbert, On the classification of the Banach spaces whose duals are $L_{1}$ spaces, J. Functional Analysis (to appear).

5. E. Michael and A. Pełczyński, Separable Banach spaces which admit $l_{n}^{\infty}$ approximations, Israel J. Math. 4 (1966), 189-198.

6. Z. Semadeni, Free compact sets, Bull. Acad. Polon. Sci. Sér Sci. Math. Astronom. Phys. 13 (1964), 141-146.

University of California, Berkeley 